\title{
Nutrient intakes and dysglycaemia in populations of West African origin
}

\author{
Simon G. Anderson ${ }^{1,2 *}$, Novie Younger ${ }^{2}$, Adrian H. Heald ${ }^{3}$, Marshall K. Tulloch-Reid ${ }^{2}$,
} Wiyumile P. Simukonda ${ }^{4}$, Jean-Claude Mbanya ${ }^{5}$, Maria D. Jackson ${ }^{6}$, Beverley Balkau ${ }^{7}$, Sangita Sharma ${ }^{8}$, Agatha Tanya ${ }^{9}$, Terrence Forrester ${ }^{2}$, Rainford Wilks ${ }^{2}$ and J. Kennedy Cruickshank ${ }^{1}$

${ }^{1}$ Cardiovascular Sciences Research Group, Core Technology Facility (3rd Floor), University of Manchester Medical School, 46 Grafton Street, Manchester M13 9NT, UK

${ }^{2}$ Tropical Medicine Research Institute, University of the West Indies, Mona, Jamaica

${ }^{3}$ Endocrine Sciences Research Group, University of Manchester, Manchester, UK

${ }^{4}$ School of Postgraduate Medical and Dental Education, Cardiff University, Cardiff, UK

${ }^{5}$ Department of Internal Medicine, University of Yaoundé I, Yaoundé, Cameroon

${ }^{6}$ Department of Community Health and Psychiatry, University of the West Indies, Mona, Jamaica

${ }^{7}$ INSERM U21, Paris, France

${ }^{8}$ Department of Medicine, 1-126 Li Ka Shing Centre for Health Research Innovation, University of Alberta, Canada

${ }^{9}$ Department of Public Health, Faculty of Medicine and Biomedical Sciences, University of Yaounde I, Yaounde, Cameroon

(Received 2 February 2010 - Revised 20 June 2010 - Accepted 2 August 2010)

\section{Abstract}

Examining the relationship between glucose intolerance and dietary intake in genetically similar populations with different dietary patterns and rates of type 2 diabetes may provide important insights into the role of diet in the pathogenesis of this disease. The objective of the present study was to assess the relationship between dietary variables and dysglycaemia/type 2 diabetes among three populations of African origin. The study design consists of a cross-sectional study of men and women of African descent aged 24-74 years from Cameroon ( $n$ 1790), Jamaica ( $n$ 857) and Manchester, UK ( $n$ 258) who were not known to have diabetes. Each participant had anthropometric measurements and underwent a $2 \mathrm{~h} 75 \mathrm{~g}$ oral glucose tolerance test. Habitual dietary intake was estimated with quantitative FFQ, developed specifically for each country. The age-adjusted prevalence of undiagnosed type 2 diabetes in Cameroon was low (1.1\%), but it was higher in Jamaica (11.6\%) and the UK (12.6\%). Adjusted generalised linear and latent mixed models used to obtain OR indicated that each $1.0 \%$ increment in energy from protein, total fat and saturated fats significantly increased the odds of type 2 diabetes by 9 (95\% CI 1.02, 1.16)\%, 5 (95\% CI, 1.01, 1.08) \% and 16 (95\% CI 1.08, 1.25)\%, respectively. A $1 \%$ increase in energy from carbohydrates and a $0 \cdot 1$ unit increment in the PUFA:SFA ratio were associated with significantly reduced odds of type 2 diabetes. The results show independent effects of dietary factors on hyperglycaemia in African origin populations. Whether modifying intake of specific macronutrients helps diabetes prevention needs testing in randomised trials.

Key words: Dysglycaemia: Type 2 diabetes: Nutrient intake: West Africans

Type 2 diabetes results from the interplay of genetic $^{(1,2)}$ and environmental factors ${ }^{(3,4)}$, which influence the dysregulation of a number of pathways ${ }^{(5-8)}$. Differences in the prevalence and incidence of type 2 diabetes between migrant and non-migrant populations may provide important information on the role of environmental factors such as dietary composition ${ }^{(9,10)}$ and physical activity ${ }^{(11)}$ in this disease while partially controlling for the effects of some genetic factors ${ }^{(12)}$. Type 2 diabetes results in multiple microvascular and macrovascular complications that are often fatal and affect a disproportionate number of persons of African origin in developed countries. Additionally, macrovascular complications from diabetes are the leading cause of death among black populations in developing countries $^{(13,14)}$. The identification of modifiable dietary risk factors for diabetes in the African Diaspora along with evidence from studies on lifestyle modification ${ }^{(15-17)}$ should help to reduce the burden of this disease worldwide.

Previously reported findings from the present study population indicated that the habitual diet in rural

Abbreviations: IFG, impaired fasting glucose; IGT, impaired glucose tolerance; P:S, PUFA:SFA; QFFQ, quantitative FFQ.

*Corresponding author: Dr S. G. Anderson, fax +44 161275 1183, email simon.anderson@manchester.ac.uk 
Cameroon contains more fat and alcohol than the diet in urban Cameroon ${ }^{(18,19)}$. Higher levels of physical activity in the rural subsistence farming community may explain the lower levels of obesity despite higher energy consumption. In addition to the differences in dietary patterns, there is variation in rates of glucose intolerance in these populations. Our earlier report described a gradation in the prevalence of diabetes in men and women with the lowest rates in rural and urban Cameroon ( 0.8 and $2.0 \%$, respectively), Jamaica having an intermediate prevalence ( $8.5 \%)$ and African-Caribbeans living in the Manchester, UK, having the highest rates (14.6\%), with the latter two sites additionally having more previously identified cases ${ }^{(12)}$.

The present study examines whether intakes of carbohydrate, protein and dietary fat assessed from carefully developed, culture-specific quantitative FFQ (QFFQ) are associated with glucose intolerance in a representative sample of people of African origin from urban Cameroon, rural Cameroon, Jamaica and Caribbean (mainly Jamaican) migrants living in inner-city Manchester, $\mathrm{UK}^{(19-23)}$.

\section{Experimental methods}

\section{Study population}

The present study was part of an international collaboration that assessed nutritional intakes, prevalence of diabetes, hypertension and their risk factors in African origin populations $^{(12,24,25)}$. Samples were recruited from Cameroon (urban and rural), Jamaica (Spanish Town) and the United Kingdom (Manchester). In Cameroon and Jamaica, communities ranging in size from 10000 to 40000 adults were sampled according to the probability proportionate to size' method with equal proportions of men and women in four age categories (25-34, 35-44, 45-54 and 55-74 years). Random samples stratified by age decade and sex were taken from population registers in inner-city Manchester where most African-Caribbeans live. Further details on the sampling scheme have been previously described ${ }^{(19,26,27)}$. Sampling methods, especially in Jamaica, were co-standardised with the International Collaborative Study on Hypertension in Blacks study, which ran contiguously with this, and all sites shared blood pressure measurement standardisation ${ }^{(24,25,28)}$.

\section{Eligibility criteria}

People of African origin were recruited if they met at least two of the following criteria: (a) ancestry - at least three grandparents should be of west African origin, (b) observed ethnicity - black African origin - a subjective assessment by the interviewer and (c) self-assigned ethnicity - black African origin. Pregnant women were excluded. The response rates in urban and rural Cameroon were 95 and 98\%, respectively, 62\% in Jamaica and $80 \%$ in the UK. In the UK group, about $73 \%$ were of Jamaican origin and the remainder from other eastern Caribbean territories. The study was approved by national and local ethics committees.

\section{Procedures}

The methodology for the cross-site standardisation of procedures and for determining glucose intolerance has been previously published ${ }^{(12)}$. A $75 \mathrm{~g}$ oral glucose tolerance test was performed on each subject. The 2006 WHO criteria were used to classify subjects according to their glucose tolerance status (normal: fasting plasma glucose $<6.1 \mathrm{mmol} / 1$ and a $2 \mathrm{~h}$ plasma glucose ( $2 \mathrm{~h}$ postprandial glucose) $<7.8 \mathrm{mmol} / \mathrm{l}$; impaired glucose regulation including impaired fasting glucose (IFG) (fasting plasma glucose $\geq 6.1 \mathrm{mmol} / \mathrm{l}$ and $<7.0 \mathrm{mmol} / \mathrm{l}$ ) and impaired glucose tolerance (IGT) ( $2 \mathrm{~h}$ plasma glucose $\geq 7.8 \mathrm{mmol} / \mathrm{l}$ but $<11.0 \mathrm{mmol} / \mathrm{l}$ ); diabetes: fasting plasma glucose $\geq 7 \cdot 0$ $\mathrm{mmol} / \mathrm{l}$ or $2 \mathrm{~h}$ postprandial glucose $\geq 11 \cdot 1 \mathrm{mmol} / \mathrm{l}$ ). Sixty-four subjects from all sites reported that they were on treatment for diabetes and were excluded from further analyses. Information on demographic and socioeconomic factors and smoking was obtained by questionnaire. The variables used here were age, education level, marital status, smoking and employment status.

\section{Dietary assessment}

The strategy for the development of a dietary assessment tool used to determine food and nutrient intakes has been reported previously ${ }^{(21,29-31)}$. In each country, an interviewer-administered QFFQ was developed to assess habitual nutrient intake during the previous 12 months and included those foods contributing at least $90 \%$ of total energy, fat, carbohydrate and protein intakes. Interviewers used local utensils, especially prepared wooden food models, and cutlery to help subjects to describe their own portion size.

For Cameroon, the questionnaire included seventy-six food items, and the variation in consumption of foods between the wet and dry seasons was ascertained. The Jamaican questionnaire included seventy foods and drinks. The UK questionnaire included 108 food items from the Caribbean as well as from Europe. To calculate the nutrient composition of the habitual diet, several food tables ${ }^{(32-34)}$ and the nutritional analysis package, Microdiet ${ }^{(35)}$, were used. Since these tables did not cover all the typical dishes eaten in Cameroon and the UK, dishes were prepared by local people, and all the contents and the final cooked dish were weighed by trained field workers to determine the nutrient composition of the dishes $^{(20)}$.

The UK questionnaire was calibrated against $24 \mathrm{~h}$ recalls and $4 \mathrm{~d}$ weighed intake in a subsample of the total population. Each 'calibrating' method measures different 
short-term time periods to the habitual estimate from the QFFQ. Spearman rank correlation coefficients ranged from 0.38 for protein intake to 0.62 for carbohydrate intake when compared with the $4 \mathrm{~d}$ weighed intake and from 0.38 for fat intake to 0.50 for energy intake when compared with the $24 \mathrm{~h}$ recall. When the QFFQ was compared with the $4 \mathrm{~d}$ weighed record, the diet of $39 \%$ of participants fell in identical quartiles and $44 \%$ were in adjacent quartiles, and no participants were grossly misclassified, showing reasonable comparison of these different methods. In Jamaica, the QFFQ responses were compared with twelve $24 \mathrm{~h}$ recalls conducted throughout the study period. Correlation coefficients (Pearson and intra-class) varied between 0.42 for retinol and 0.71 for carbohydrate, with most values falling between 0.50 and 0.60. The reproducibility of the QFFQ was tested in a subsample of 118 subjects, and Pearson's correlation coefficients between protein, fat and carbohydrate intakes from the first and the second measurement were 0.62, 0.67 and 0.69 , respectively ${ }^{(21)}$.

\section{Statistical methods}

The data were analysed using Stata version 10.2 (StataCorp, College Station, TX, USA). An a priori exclusion criterion was used for total energy intakes. Participants with energy intakes more extreme than 2 SD above or below the mean total energy were therefore excluded. The final sample included $n 991$ (urban Cameroon), $n 658$ (rural Cameroon), $n 847$ (Jamaica) and $n 246$ (UK). As diabetes rates in both Cameroonian settings were low, data from rural and urban Cameroon were combined for some analyses in this report. Anthropometric and metabolic data are expressed as arithmetic means with 95\% CI. Initially, comparisons were made across the three population groups using Kruskal-Wallis, $\chi^{2}$ and ANOVA tests.

Nutrient intakes were adjusted for total energy by computing residuals from regression analyses, with energy intake as the independent variable and nutrient intake as the dependent variable ${ }^{(12,36)}$. Residuals were added to the expected nutrient value for the mean energy intake of the sample to obtain a score adjusted for the average energy intake. Variance components models, with fasting glucose and $2 \mathrm{~h}$ postprandial glucose as outcome variables, were developed to estimate the intra-cluster correlation and to determine relevance of linear mixed models for these analyses. With each of the four sites regarded as a cluster, random effects generalised linear mixed models (with the identity link) were used to determine the nature of the relationship between the respective nutrient scores and each of fasting glucose and $2 \mathrm{~h}$ postprandial plasma glucose levels. The same methodology was used to determine whether the country of origin was an effect modifier of the respective relationships. The results presented are country-specific and cross-country estimates of the increment in the respective outcome variables in response to $0 \cdot 1$ unit increments in the PUFA:SFA (P:S) ratio or 1 unit increments in percentage energy from the respective nutrients. The estimates were adjusted for age, sex, BMI, country of origin, level of education attained, smoking status and tertiles of alcohol consumed.

Mixed effects logistic regression models (generalised linear mixed models with the logit link) yielded OR adjusted for age, sex, country, BMI, level of education attained, smoking status and tertiles of alcohol consumed for the association of glucose tolerance status - IFG/IGT or newly detected type 2 diabetes - with previously mentioned incremental changes in the respective nutrient scores. The models resulted in cross-country and country-specific estimates of the relative odds of having either IGT/IFG or type 2 diabetes $v$. being normoglycaemic. The models that provided country-specific estimates also indicated whether there was an interaction of country with nutrient intakes in the effect of the latter on the odds of IGT/IFG or type 2 diabetes. As not all participants had all measurements due to missing data, numbers varied between analyses.

\section{Results}

Men and women were youngest in Cameroon and oldest in Manchester, UK (Table 1). BMI was highest in the UK for both sexes and lowest in Cameroon, while waist:hip ratios were highest in Manchester men. Overall, there was a cross-site gradient of obesity $\left(\mathrm{BMI} \geq 30 \mathrm{~kg} / \mathrm{m}^{2}\right)$ prevalence (Cameroon 16.1\%; Jamaica 31.2\% and UK $\left.35 \cdot 1 \% ; \chi^{2} 59 \cdot 1, P=0 \cdot 0001\right)$; however, male obesity rates were lowest in Jamaica and Cameroon and nearly four times higher in the UK population (UK 20.4\%; $\chi^{2} 24.5$, $P=0.0001$ ). Smoking prevalence was highest in men and women from Manchester. Fasting plasma glucose levels were lowest in Cameroonian participants (Table 1).

Table 2 presents the details of energy intake and the percentage contribution of macronutrients by country. Crude-reported total energy intake was highest in Cameroon men and women and lowest in the UK. Percentage energy from total fat was about $10 \%$ higher in Cameroon (mainly from staple palm nut oil) than either Jamaican or UK men or women. However, Jamaicans had the lowest percentage energy from PUFA and SFA. P:S ratios in women were significantly higher in participants from Manchester but were similar in Cameroon and Jamaica.

Additionally, for both men and women, Table 2 indicates that percentage energy from protein in the UK and Jamaica was significantly higher $(P=0.0001)$ than in Cameroon. More than $58 \%$ of the reported total energy intake came from carbohydrate in Jamaicans; this was only slightly less at about $52-55 \%$ of total energy in Manchester and less than $50 \%$ of total energy in Cameroon. Daily intake of dietary fibre was significantly higher in Cameroon compared to the other populations. 
Table 1. Anthropometric, demographic and metabolic features of participants by country* (Mean values and $95 \%$ confidence intervals or percentages)

\begin{tabular}{|c|c|c|c|c|c|c|c|}
\hline & \multicolumn{2}{|c|}{ Cameroon } & \multicolumn{2}{|c|}{ Jamaica } & \multicolumn{2}{|c|}{ Manchester } & \multirow[b]{2}{*}{$P$} \\
\hline & Mean/\% & $95 \% \mathrm{Cl}$ & Mean/\% & $95 \% \mathrm{Cl}$ & Mean/\% & $95 \% \mathrm{Cl}$ & \\
\hline$n$ & \multicolumn{2}{|c|}{959} & \multicolumn{2}{|c|}{507} & \multicolumn{2}{|c|}{146} & \\
\hline \multicolumn{8}{|l|}{ Women } \\
\hline Age (years) & $41 \cdot 2$ & $40 \cdot 4,42 \cdot 0$ & $45 \cdot 8$ & $44 \cdot 7,46 \cdot 9$ & 48.6 & $46 \cdot 6,50 \cdot 6$ & $F=38.4 ; P<0.0001$ \\
\hline $\operatorname{BMI}\left(\mathrm{kg} / \mathrm{m}^{2}\right)$ & $25 \cdot 1$ & $24 \cdot 7,25 \cdot 4$ & $27 \cdot 6$ & $27 \cdot 1,28 \cdot 1$ & $28 \cdot 7$ & $27 \cdot 8,29 \cdot 6$ & $F=51.8 ; P<0.0001$ \\
\hline Waist:hip ratio & 0.83 & $0.82,0.84$ & 0.79 & $0.79,0.80$ & 0.82 & $0.81,0.84$ & $F=19.6 ; P<0.0001$ \\
\hline Fasting glucose $(\mathrm{mmol} / \mathrm{l})$ & 4.2 & $4 \cdot 1,4 \cdot 3$ & 5.6 & $5 \cdot 4,5 \cdot 7$ & $5 \cdot 5$ & $5 \cdot 2,5 \cdot 9$ & $F=92.8 ; P<0.0001$ \\
\hline $2 \mathrm{~h}$ Glucose $(\mathrm{mmol} / \mathrm{l})$ & $5 \cdot 0$ & $4 \cdot 9,5 \cdot 2$ & $7 \cdot 6$ & $7 \cdot 4,7 \cdot 9$ & $5 \cdot 9$ & $5 \cdot 3,6 \cdot 6$ & $F=139.6 ; P<0.0001$ \\
\hline Alcohol consumed $(\mathrm{g} / \mathrm{d})$ & $7 \cdot 6$ & $6.7,8.4$ & $1 \cdot 1$ & $0.7,1.4$ & 3.0 & $2 \cdot 2,3.7$ & $\chi^{2} 302.0 ; P=0.0001$ \\
\hline \multicolumn{8}{|l|}{ Glucose tolerance $(\%)$} \\
\hline Normoglycaemic & 97 & & 67 & & 76 & & \\
\hline IGR & 2 & & 19 & & 13 & & $\chi^{2} 250.1 ; P<0.0001$ \\
\hline Type 2 diabetes & 1 & & 14 & & 10 & & \\
\hline \multicolumn{8}{|l|}{ Smoking status (\%) } \\
\hline Non-smoker & $96 \cdot 1$ & & $80 \cdot 1$ & & 75 & & \\
\hline Ex-smoker & 1.6 & & 8.5 & & 8.2 & & $\chi^{2} 123.9 ; P<0.0001$ \\
\hline Current smoker & $2 \cdot 3$ & & 11.4 & & $17 \cdot 1$ & & \\
\hline \multicolumn{8}{|l|}{ Education (\%) } \\
\hline Primary or less & 47 & & 69 & & 2 & & \\
\hline Secondary or more & 28 & & 23 & & 72 & & $\chi^{2} 253 ; P<0.0001$ \\
\hline College or more & 25 & & 8 & & 26 & & \\
\hline$n$ & \multirow{2}{*}{\multicolumn{2}{|c|}{690}} & \multirow{2}{*}{\multicolumn{2}{|c|}{340}} & \multirow{2}{*}{\multicolumn{2}{|c|}{100}} & \\
\hline Men & & & & & & & \\
\hline Age (years) & $41 \cdot 2$ & $40 \cdot 3,42 \cdot 2$ & $45 \cdot 8$ & $44 \cdot 4,47 \cdot 1$ & $52 \cdot 9$ & $50 \cdot 4,55 \cdot 4$ & $\mathrm{~F}=42.8 ; P<0.0001$ \\
\hline BMI $\left(\mathrm{kg} / \mathrm{m}^{2}\right)$ & 23.7 & $23 \cdot 4,24 \cdot 0$ & 23.5 & $23 \cdot 1,23 \cdot 9$ & $27 \cdot 1$ & $26 \cdot 4,27 \cdot 9$ & $F=37.3 ; P<0.0001$ \\
\hline Waist:hip ratio & 0.87 & $0.86,0.88$ & 0.84 & $0.83,0.84$ & 0.91 & $0.89,0.92$ & $F=40.1 ; P<0.0001$ \\
\hline Fasting glucose $(\mathrm{mmol} / \mathrm{l})$ & $4 \cdot 2$ & $4 \cdot 1,4 \cdot 3$ & $5 \cdot 4$ & $5 \cdot 2,5 \cdot 6$ & $5 \cdot 7$ & $5 \cdot 2,6 \cdot 1$ & $F=59.5 ; P<0.0001$ \\
\hline $2 \mathrm{~h}$ Glucose $(\mathrm{mmol} / \mathrm{l})$ & $5 \cdot 2$ & $5 \cdot 0,5 \cdot 4$ & 6.4 & $6 \cdot 2,6 \cdot 7$ & $7 \cdot 0$ & $6 \cdot 3,7 \cdot 7$ & $F=31.9 ; P<0.0001$ \\
\hline Alcohol consumed $(\mathrm{g} / \mathrm{d})$ & $19 \cdot 4$ & $17 \cdot 4,21 \cdot 3$ & $10 \cdot 0$ & $8 \cdot 2,11 \cdot 8$ & 7.5 & $5 \cdot 4,9 \cdot 6$ & $\chi^{2} 66 \cdot 3 ; P=0.0001$ \\
\hline \multicolumn{8}{|l|}{ Glucose tolerance $(\%)$} \\
\hline Normoglycaemic & 95 & & 77 & & 72 & & \\
\hline $\mathrm{IGR}^{\star}$ & 4 & & 15 & & 13 & & $\chi^{2} 95.9 ; P<0.0001$ \\
\hline Type 2 diabetes & 1 & & 8 & & 15 & & \\
\hline \multicolumn{8}{|l|}{ Smoking status (\%) } \\
\hline Non-smoker & 67.5 & & $37 \cdot 1$ & & 43.4 & & \\
\hline Ex-smoker & 11.8 & & $22 \cdot 6$ & & $25 \cdot 3$ & & $\chi^{2} 94.4 ; P<0.001$ \\
\hline Current smoker & $20 \cdot 7$ & & $40 \cdot 3$ & & $31 \cdot 3$ & & \\
\hline \multicolumn{8}{|l|}{ Education (\%) } \\
\hline Primary or less & 30 & & 65 & & 5 & & \\
\hline Secondary or more & 25 & & 28 & & 67 & & $\chi^{2} 245 ; P<0.0001$ \\
\hline College or more & 45 & & 7 & & 28 & & \\
\hline
\end{tabular}

IGR, impaired glucose regulation for impaired fasting glucose/impaired glucose tolerance combined.

* Comparisons were made using ANOVA or Kruskal-Wallis test for continuous variables or $\chi^{2}$ test for categorical variables.

\section{Relationship between nutrient intake and diabetes status}

To account for correlation between responses within each of the four sites, mixed effects logistic regression models were used to obtain OR for the association of different nutrients with occurrence of IFG/IGT and type 2 diabetes. The OR for the association of different energy-adjusted nutrients with the presence of IFG/IGT and type 2 diabetes are shown in Table 3. The results indicate, for all sites combined, that a $1 \%$ increment in energy from protein, total fat and saturated fats significantly increased the odds of type 2 diabetes by 10, 4 and 15\%, respectively. Conversely, each $1 \%$ increment in energy from carbohydrates and a $0 \cdot 1$ unit increment in P:S ratio significantly reduced the odds of having type 2 diabetes by 4 and $12 \%$. When these crude estimates were adjusted for age, sex, BMI and country (Cameroon, Jamaica and UK), the significant association of percentage energy from protein, carbohydrate, total fat and saturated fat was retained. In separate models adjusting for smoking, the level of educational attainment and tertiles of alcohol consumption did not modify the nature of the respective relationships between the nutrients and the outcomes as shown in Table 3. We found no evidence of an association between IFG/IGT with any of the nutrients.

\section{Relationship between nutrient intake and diabetes status by site}

Mixed effects logistic regression analyses were also used to assess whether the relationship between nutrient intakes and the odds of glucose tolerance status was modified by country. Data presented in Table 4 suggest that country of origin interacts with percentage energy from 
Table 2. Age-adjusted energy intakes and percentage contribution of macronutrient intakes by country ${ }^{\star}$ (Mean values and $95 \%$ confidence intervals)

\begin{tabular}{|c|c|c|c|c|c|c|c|}
\hline & \multicolumn{2}{|c|}{ Cameroon } & \multicolumn{2}{|c|}{ Jamaica } & \multicolumn{2}{|c|}{ Manchester } & \multirow[b]{2}{*}{$P$} \\
\hline & Mean & $95 \% \mathrm{Cl}$ & Mean & $95 \% \mathrm{Cl}$ & Mean & $95 \% \mathrm{Cl}$ & \\
\hline$n$ & \multicolumn{2}{|r|}{959} & \multicolumn{2}{|c|}{507} & \multicolumn{2}{|r|}{146} & \\
\hline \multicolumn{8}{|l|}{ Women } \\
\hline Total energy intake $(\mathrm{MJ} / 24 \mathrm{~h})^{\star}$ & $14 \cdot 4$ & $14 \cdot 1,14 \cdot 7$ & $9 \cdot 7$ & $9 \cdot 3,10 \cdot 1$ & $8 \cdot 0$ & $7 \cdot 2,8 \cdot 7$ & $F=259.8 ; P<0.0001$ \\
\hline Protein (\% E) & $10 \cdot 0$ & $9 \cdot 9,10 \cdot 1$ & $12 \cdot 4$ & $12 \cdot 2,12 \cdot 6$ & $13 \cdot 7$ & $13 \cdot 3,14 \cdot 1$ & $F=489.4 ; P<0.0001$ \\
\hline Carbohydrate (\% E) & $48 \cdot 7$ & $48 \cdot 1,49 \cdot 3$ & $58 \cdot 0$ & $57 \cdot 2,58,8$ & $52 \cdot 9$ & $51 \cdot 5,54.4$ & $F=177.1 ; P<0.0001$ \\
\hline Total fat $(\% \mathrm{E})$ & 41.9 & $41 \cdot 4,42 \cdot 3$ & $32 \cdot 8$ & $32 \cdot 1,33 \cdot 4$ & $36 \cdot 1$ & $34.9,37.4$ & $F=235.2 ; P<0.0001$ \\
\hline PUFA (\% E) & $5 \cdot 8$ & $5.7,5.9$ & 3.4 & $3.2,3.5$ & $6 \cdot 7$ & $6 \cdot 5,6 \cdot 9$ & $F=711.1 ; P<0.0001$ \\
\hline SFA (\% E) & 13.9 & $13 \cdot 7,14 \cdot 1$ & $8 \cdot 7$ & $8 \cdot 4,9 \cdot 0$ & $13 \cdot 0$ & $12 \cdot 5,13 \cdot 6$ & $F=378.3 ; P<0.0001$ \\
\hline$P: S$ ratio & 0.43 & $0.43,0.44$ & 0.41 & $0.39,0.42$ & 0.54 & $0.52,0.56$ & $F=64.33 ; P<0.0001$ \\
\hline Fibre (g) & $36 \cdot 4$ & $30 \cdot 4,37 \cdot 3$ & $23 \cdot 4$ & $31 \cdot 0,24 \cdot 7$ & $22 \cdot 5$ & $20 \cdot 1,24 \cdot 9$ & $F=156.6 ; P<0.0001$ \\
\hline$n$ & \multirow{2}{*}{\multicolumn{2}{|c|}{690}} & \multirow{2}{*}{\multicolumn{2}{|c|}{340}} & \multirow{2}{*}{\multicolumn{2}{|c|}{100}} & \\
\hline Men & & & & & & & \\
\hline Total energy intake* (MJ/24 h) & $15 \cdot 7$ & $15 \cdot 4,16 \cdot 1$ & $12 \cdot 4$ & $11 \cdot 9,12 \cdot 8$ & $10 \cdot 0$ & $9 \cdot 1,10 \cdot 9$ & $F=94.3 ; P<0.0001$ \\
\hline Protein (\% E) & $10 \cdot 2$ & $10 \cdot 1,10 \cdot 4$ & $13 \cdot 2$ & $12 \cdot 9,13 \cdot 4$ & 14.4 & $14 \cdot 0,14 \cdot 8$ & $F=317.6 ; P<0.0001$ \\
\hline Carbohydrate (\% E) & $47 \cdot 7$ & $46 \cdot 9,48 \cdot 3$ & 58.9 & $57.9,60 \cdot 0$ & $54 \cdot 8$ & $52 \cdot 9,56 \cdot 8$ & $F=153.2 ; P<0.0001$ \\
\hline Total fat $(\% \mathrm{E})$ & $40 \cdot 9$ & $40 \cdot 2,41 \cdot 4$ & $31 \cdot 8$ & $31 \cdot 0,32 \cdot 7$ & $34 \cdot 7$ & $33 \cdot 1,36 \cdot 3$ & $F=150.8 ; P<0.0001$ \\
\hline PUFA (\% E) & 5.5 & $5 \cdot 4,5 \cdot 6$ & 3.6 & $3.5,3.7$ & $6 \cdot 0$ & $5 \cdot 7,6 \cdot 3$ & $F=279.3 ; P<0.0001$ \\
\hline SFA (\% E) & $13 \cdot 3$ & $13 \cdot 1,13 \cdot 6$ & $7 \cdot 7$ & $7 \cdot 3,8 \cdot 0$ & $12 \cdot 4$ & $11 \cdot 7,13 \cdot 0$ & $F=314.5 ; P<0.0001$ \\
\hline $\mathrm{P}: \mathrm{S}$ ratio & 0.43 & $0.42,0.44$ & 0.50 & $0.49,0.52$ & 0.52 & $0.49,0.54$ & $F=40.2 ; P<0.0001$ \\
\hline Fibre $(g)$ & $37 \cdot 3$ & $36 \cdot 1,38 \cdot 4$ & $30 \cdot 6$ & $28 \cdot 9,32 \cdot 2$ & 28.9 & $25 \cdot 8,31 \cdot 9$ & $F=27.5 ; P<0.0001$ \\
\hline
\end{tabular}

$\% \mathrm{E}$, energy percentage; P:S, PUFA:SFA.

* Comparisons were made using ANOVA for continuous variables.

carbohydrate in its effect on glucose tolerance state. As such, higher percentage energy from carbohydrates significantly reduced the odds of IFG/IGT in persons from Cameroon. After adjusting for age, sex and BMI, higher values of percentage energy from protein, total fat and saturated fat significantly increased the odds of type 2 diabetes in Jamaicans. In a separate model, the independent relationship between the percentage energy from protein or fats and the odds of having type 2 diabetes was not modified when smoking status or tertiles of alcohol consumption were added to the multivariate models. Increasing percentage energy from PUFA significantly increased the odds for type 2 diabetes in the Cameroonian participants. A higher P:S ratio was associated with reduced odds of type 2 diabetes in Jamaicans only.

\section{Relationship between nutrient intake and glucose as a continuous variable}

Generalised linear and latent mixed models (with the identity link function) were used to determine the nature of the relationship between percentage energy from different macronutrients, P:S ratio and glucose levels. There was a positive association between percentage energy intakes from protein and SFA with both fasting glucose and $2 \mathrm{~h}$ glucose (Table 5). Unadjusted P:S ratio was borderline associated with fasting glucose $(P=0.05)$. In addition, a statistically significant negative relationship of the P:S ratio with fasting glucose $(P=0.017)$ and $2 \mathrm{~h}$ postprandial glucose $(P=0.001)$ was found in both the unadjusted and adjusted analyses (Table 5). The results shown in Table 5

Table 3. Unadjusted and adjusted country-specific point estimates of the relative odds for impaired fasting glucose/impaired glucose tolerance (IFG/IGT) or type 2 diabetes associated with incremental changes in nutrient intakes as produced by generalised linear mixed models for categorical outcomes

(Odds ratio and $95 \%$ confidence intervals)

\begin{tabular}{|c|c|c|c|c|c|c|c|c|}
\hline \multirow[b]{3}{*}{ Nutrients } & \multicolumn{4}{|c|}{ Unadjusted OR† } & \multicolumn{4}{|c|}{ Adjusted OR†‡ } \\
\hline & \multicolumn{2}{|c|}{ IFG/IGT } & \multicolumn{2}{|c|}{ Type 2 diabetes } & \multicolumn{2}{|c|}{ IFG/IGT } & \multicolumn{2}{|c|}{ Type 2 diabetes } \\
\hline & OR & $95 \% \mathrm{Cl}$ & OR & $95 \% \mathrm{Cl}$ & OR & $95 \% \mathrm{Cl}$ & OR & $95 \% \mathrm{Cl}$ \\
\hline Protein (per unit \% E) & 1.00 & $0.94,1.06$ & $1 \cdot 10^{*}$ & $1 \cdot 03,1 \cdot 17$ & 0.99 & $0.93,1.06$ & $1.09^{*}$ & $1.02,1.16$ \\
\hline Carbohydrate (per unit \% E) & 0.99 & $0.97,1.07$ & $0.96^{\star}$ & $0.94,0.98$ & 0.99 & $0.97,1.01$ & $0.97^{\star}$ & $0.94,0.99$ \\
\hline Total fat (per unit \% E) & 1.01 & $0.99,1.04$ & $1.04^{\star}$ & $1.01,1.08$ & 1.01 & $0.99,1.04$ & $1.05^{\star}$ & $1.01,1.08$ \\
\hline SFA (per unit \% E) & 1.05 & $0.99,1.11$ & $1 \cdot 15^{\star}$ & $1.07,1.22$ & 1.05 & $0.99,1.11$ & $1 \cdot 16^{*}$ & $1.08,1.25$ \\
\hline PUFA (per unit \% E) & 1.02 & $0.91,1.15$ & 1.06 & $0.92,1.22$ & 1.05 & $0.93,1.18$ & $1 \cdot 12^{*}$ & $0.96,1.30$ \\
\hline $\mathrm{P}: \mathrm{S}$ ratio (per unit) & 0.93 & $0.84,1.03$ & $0.88^{*}$ & $0.77,0.99$ & 0.96 & $0.86,1.06$ & 0.90 & $0.79,1.01$ \\
\hline
\end{tabular}

$\% E$, energy percentage; $\mathrm{P}: \mathrm{S}, \mathrm{PUFA}$ :SFA.

${ }^{\star} P<0.05$.

† Reference category was normoglycaemia.

$\ddagger$ Estimates adjusted for age, sex, country and BMI. 
Table 4. Unadjusted and adjusted country-specific point estimates of the relative odds of impaired fasting glucose and/or impaired glucose tolerance (IFG/IGT) or type 2 diabetes associated with incremental changes in nutrient intakes as produced by generalised linear mixed models for categorical outcomes

(Odds ratios and $95 \%$ confidence intervals)

\begin{tabular}{|c|c|c|c|c|c|c|c|c|}
\hline & \multicolumn{4}{|c|}{ Unadjusted OR } & \multicolumn{4}{|c|}{ Adjusted OR $†$} \\
\hline & \multicolumn{2}{|c|}{ IFG/IGT } & \multicolumn{2}{|c|}{ T2DM } & \multicolumn{2}{|c|}{ IFG/IGT } & \multicolumn{2}{|c|}{ T2DM } \\
\hline & OR & $95 \% \mathrm{Cl}$ & OR & $95 \% \mathrm{Cl}$ & OR & $95 \% \mathrm{Cl}$ & OR & $95 \% \mathrm{Cl}$ \\
\hline \multicolumn{9}{|l|}{ Cameroon } \\
\hline Protein (per unit \% E) & 0.90 & $0.77,1.06$ & 1.03 & $0.81,1.33$ & 0.95 & $0.81,1.11$ & 1.09 & $0.85,1.40$ \\
\hline Carbohydrate (per unit \% E) & $0.96^{*}$ & $0.93,0.99$ & 0.98 & $0.94,1.03$ & 0.97 & $0.94,1.00$ & 0.99 & $0.95,1.03$ \\
\hline Total fat (per unit \% E) & 1.03 & $0.99,1.07$ & 1.03 & $0.98,1.09$ & 1.03 & $0.99,1.06$ & 1.03 & $0.97,1.08$ \\
\hline SFA (per unit \% E) & $1 \cdot 07^{\star}$ & $1 \cdot 00,1 \cdot 16$ & $1 \cdot 13^{*}$ & $1 \cdot 00,1 \cdot 28$ & 1.06 & $0.98,1 \cdot 14$ & $1 \cdot 12$ & $0.99,1.26$ \\
\hline PUFA (per unit \% E) & 1.16 & $0.93,1.45$ & $1.44^{\star}$ & $1.01,2.04$ & $1 \cdot 10$ & $0.89,1.37$ & 1.33 & $0.94,1.87$ \\
\hline $\mathrm{P}: \mathrm{S}$ ratio (per unit) & 0.73 & $0.46,1.17$ & 0.87 & $0.47,1.61$ & 0.78 & $0.51,1.18$ & 0.80 & $0.43,1.50$ \\
\hline \multicolumn{9}{|l|}{ Jamaica } \\
\hline Protein (per unit \% E) & 0.99 & $0.92,1.06$ & $1.09^{*}$ & $1 \cdot 02,1 \cdot 17$ & 0.99 & $0.93,1.06$ & $1.08^{\star}$ & $1.01,1 \cdot 16$ \\
\hline Carbohydrate (per unit \% E) & 1.00 & $0.98,1.03$ & 0.95 & $0.93,0.98$ & 1.00 & $0.98,1.03$ & $0.96^{\star}$ & $0.93,0.99$ \\
\hline Total fat (per unit \% E) & 0.99 & $0.96,1.03$ & $1.05^{*}$ & $1.01,1.09$ & 1.00 & $0.96,1.03$ & $1.05^{*}$ & $1.01,1.09$ \\
\hline SFA (per unit \% E) & 1.04 & $0.97,1.13$ & $1 \cdot 19^{*}$ & $1.09,1.30$ & 1.05 & $0.95,1.13$ & $1 \cdot 20^{\star}$ & $1.09,1.31$ \\
\hline PUFA (per unit \% E) & 0.98 & $0.84,1.15$ & 0.97 & $0.80,1.17$ & 1.04 & $0.88,1.23$ & 1.06 & $0.87,1.30$ \\
\hline$P: S$ ratio (per unit) & 0.93 & $0.83,1.03$ & $0.86^{*}$ & $0.75,0.98$ & 0.96 & $0.85,1.07$ & 0.90 & $0.78,1.03$ \\
\hline \multicolumn{9}{|l|}{ Manchester, UK } \\
\hline Protein (per unit \% E) & 1.05 & $0.84,1.32$ & $1 \cdot 18$ & $0.93,1.51$ & 1.05 & $0.82,1.34$ & $1 \cdot 16$ & $0.89,1.52$ \\
\hline Carbohydrate (per unit \% E) & 0.99 & $0.91,1.07$ & 0.93 & $0.85,1.02$ & 0.99 & $0.91,1.08$ & 0.91 & $0.82,1.01$ \\
\hline Total fat (per unit \% E) & 1.03 & $0.93,1.13$ & 1.08 & $0.97,1.20$ & 1.02 & $0.92,1.14$ & $1 \cdot 12$ & $0.99,1.27$ \\
\hline SFA (per unit \% E) & 0.97 & $0.80,1 \cdot 19$ & 1.06 & $0.85,1.31$ & 1.00 & $0.80,1.23$ & 1.14 & $0.90,1.45$ \\
\hline PUFA (per unit \% E) & 0.97 & $0.68,1.37$ & $1 \cdot 12$ & $0.79,1.59$ & 0.96 & $0.68,1.36$ & 1.08 & $0.75,1.57$ \\
\hline$P: S$ ratio (per unit) & $1 \cdot 10$ & $0.83,1.47$ & 1.03 & $0.73,1.47$ & 1.05 & $0.79,1.40$ & 0.95 & $0.66,1.36$ \\
\hline
\end{tabular}

T2DM, type 2 diabetes mellitus; \% E, energy percentage; P:S, PUFA:SFA.

${ }^{*} P<0.05$.

$\dagger$ Estimates adjusted for age, sex and BMI.

were unchanged by adjustment for each of education, tertiles of alcohol consumption and smoking status.

\section{Relationship between nutrient intake and glucose as a continuous variable by country}

Table 6 illustrates the results of models aimed at assessing whether country of origin (nationality) modified the relationship between reported nutrient intake and glucose levels. Adjusted results demonstrated a significant direct association between percentage energy from protein and fasting glucose in Jamaicans $(0.07 \mathrm{mmol} / 1$ higher in participants whose percentage energy from protein is higher by $1 \%$ ) and participants from the UK but not in Cameroon. For the Jamaican participants, each unit increase in SFA was associated with a $0.12 \mathrm{mmol} / 1$ increase in fasting glucose $(0.22 \mathrm{mmol} / 1$ for $2 \mathrm{~h}$ glucose). There was an inverse relationship between percentage energy from carbohydrates $(\beta=-0.023)$ and fasting glucose. The percentage energy from total fat, as a $0.045 \mathrm{mmol} / 1$ increase for a 1 unit change in this nutrient index, was also observed with $2 \mathrm{~h}$ glucose but not with fasting glucose.

Table 5. Unadjusted and adjusted country-specific point estimates of the increment in glucose levels (per $1 \mathrm{mmol} / \mathrm{l}$ ) associated with incremental changes in nutrient intakes as produced by generalised linear mixed models for continuous outcomes

( $\beta$ Coefficients and $95 \%$ confidence intervals)

\begin{tabular}{|c|c|c|c|c|c|c|c|c|}
\hline \multirow[b]{3}{*}{ Nutrients $\ddagger$} & \multicolumn{4}{|c|}{ Unadjusted $\beta$ coefficients } & \multicolumn{4}{|c|}{ Adjusted $\beta$ coefficients $\dagger$} \\
\hline & \multicolumn{2}{|c|}{ Fasting glucose } & \multicolumn{2}{|c|}{$2 \mathrm{~h}$ glucose } & \multicolumn{2}{|c|}{ Fasting glucose } & \multicolumn{2}{|c|}{$2 \mathrm{~h}$ glucose } \\
\hline & $\beta$ & $95 \% \mathrm{Cl}$ & $\beta$ & $95 \% \mathrm{Cl}$ & $\beta$ & $95 \% \mathrm{Cl}$ & $\beta$ & $95 \% \mathrm{Cl}$ \\
\hline Protein (per unit \% E) & $0.05^{\star}$ & $0.02,0.09$ & $0 \cdot 09^{*}$ & $0.04,0.13$ & $0.06^{*}$ & $0.02,0.09$ & $0 \cdot 08^{\star}$ & $0.03,0.13$ \\
\hline Carbohydrate (per unit \% E) & -0.01 & $-0.01,0.001$ & $-0.01^{*}$ & $-0.03,-0.003$ & -0.005 & $-0.01,0.003$ & -0.01 & $-0.02,0.001$ \\
\hline Total fat (per unit \% E) & 0.01 & $-0.004,0.01$ & 0.01 & $-0.004,0.02$ & 0.005 & $-0.005,0.01$ & 0.01 & $-0.01,0.02$ \\
\hline SFA (per unit \% E) & $0.03^{*}$ & $0.01,0.05$ & $0.04^{*}$ & $0.01,0.07$ & $0.03^{*}$ & $0.01,0.05$ & $0.03^{*}$ & $0.002,0.06$ \\
\hline PUFA (per unit \% E) & 0.004 & $-0.05,0.06$ & -0.01 & $-0.01,0.07$ & -0.001 & $-0.06,0.05$ & -0.03 & $-0.11,0.05$ \\
\hline $\mathrm{P}: \mathrm{S}$ ratio (per unit) & $-0.07^{\star}$ & $-0.12,-0.01$ & $-0 \cdot 14^{\star}$ & $-0.22,-0.06$ & $-0.07^{\star}$ & $-0.13,-0.02$ & $-0.11^{\star}$ & $-0.19,-0.03$ \\
\hline
\end{tabular}

$\% E$, energy percentage; $P: S$, PUFA:SFA.

${ }^{*} P<0.05$.

$\dagger$ Estimates adjusted for age, sex, country and BMI.

$\ddagger$ Nutrients are per unit percentage energy (per unit \% E). 
Table 6. Unadjusted and adjusted country-specific point estimates of the increment in glucose level (fasting and $2 \mathrm{~h}$ ) associated with incremental changes in nutrient intakes as produced by generalised linear mixed models for continuous outcomes stratified by country ( $\beta$ Coefficients and $95 \%$ confidence intervals)

\begin{tabular}{|c|c|c|c|c|c|c|c|c|}
\hline & \multicolumn{4}{|c|}{ Unadjusted $\beta$ coefficients } & \multicolumn{4}{|c|}{ Adjusted $\beta$ coefficients $\dagger$} \\
\hline & \multicolumn{2}{|c|}{ Fasting glucose } & \multicolumn{2}{|c|}{$2 \mathrm{~h}$ glucose } & \multicolumn{2}{|c|}{ Fasting glucose } & \multicolumn{2}{|c|}{$2 \mathrm{~h}$ glucose } \\
\hline & $\beta$ & $95 \% \mathrm{Cl}$ & $\beta$ & $95 \% \mathrm{Cl}$ & $\beta$ & $95 \% \mathrm{Cl}$ & $\beta$ & $95 \% \mathrm{Cl}$ \\
\hline \multicolumn{9}{|l|}{ Cameroon } \\
\hline Protein (per unit \% E) & 0.01 & $-0.04,0.05$ & -0.02 & $-0.10,0.06$ & 0.02 & $-0.02,0.10$ & 0.01 & $-0.06,0.08$ \\
\hline Carbohydrate (per unit \% E) & -0.001 & $-0.01,0.001$ & -0.005 & $-0.01,0.008$ & 0.001 & $-0.01,0.01$ & -0.001 & $-0.01,0.01$ \\
\hline Total fat (per unit \% E) & 0.002 & $-0.01,0.01$ & 0.004 & $-0.01,0.02$ & 0.001 & $-0.01,0.01$ & -0.001 & $-0.02,0.01$ \\
\hline SFA (per unit \% E) & 0.01 & $-0.01,0.04$ & $0.03^{*}$ & $0.02,0.04$ & 0.01 & $-0.01,0.03$ & -0.001 & $-0.03,0.03$ \\
\hline PUFA (per unit \% E) & 0.02 & $-0.05,0.08$ & 0.03 & $-0.07,0.13$ & 0.001 & $-0.07,0.07$ & -0.02 & $-0.12,0.07$ \\
\hline$P: S$ ratio (per 0.1 unit) & -0.05 & $-0.16,0.06$ & -0.02 & $-0.20,0.16$ & -0.06 & $-0.18,0.05$ & -0.04 & $-0.20,0.13$ \\
\hline \multicolumn{9}{|l|}{ Jamaica } \\
\hline Protein (per unit \% E) & $0.07^{\star}$ & $0.03,0.12$ & $0 \cdot 14^{*}$ & $0.07,0.21$ & $0.07^{*}$ & $0.03,0.12$ & $0 \cdot 14^{*}$ & $0.07,0.20$ \\
\hline Carbohydrate (per unit \% E) & $-0.03^{*}$ & $-0.04,-0.01$ & $-0.06^{\star}$ & $-0.08,-0.03$ & $-0.02^{*}$ & $-0.04,-0.01$ & $-0.05^{\star}$ & $-0.08,-0.03$ \\
\hline Total fat (per unit \% E) & $0.02^{*}$ & $0.001,0.05$ & $0.05^{\star}$ & $0.02,0.08$ & 0.02 & $-0.001,0.04$ & $0.04^{*}$ & $0.01,0.08$ \\
\hline SFA (per unit \% E) & $0 \cdot 12^{*}$ & $0.07,0.18$ & $0 \cdot 24^{*}$ & $0.16,0.32$ & $0 \cdot 12^{*}$ & $0.07,0.17$ & $0.22^{*}$ & $0.15,0.30$ \\
\hline PUFA (per unit \% E) & -0.02 & $-0.13,0.09$ & $-0 \cdot 10$ & $-0.26,0.06$ & -0.002 & $-0.11,0.11$ & -0.04 & $-0.20,0.11$ \\
\hline$P: S$ ratio (per 0.1 unit) & $-0.09^{\star}$ & $-0.16,-0.03$ & $-0 \cdot 21^{*}$ & $-0.31,-0.11$ & $-0.08^{*}$ & $-0.15,-0.02$ & $-0 \cdot 17^{\star}$ & $-0.26,-0.07$ \\
\hline \multicolumn{9}{|l|}{ Manchester, UK } \\
\hline Protein (per unit \% E) & $0.22^{*}$ & $0.08,0.36$ & 0.15 & $-0.08,0.37$ & $0 \cdot 19^{*}$ & $0.05,0.33$ & 0.09 & $-0.13,0.31$ \\
\hline Carbohydrate (unit \% E) & -0.04 & $-0.09,0.01$ & 0.01 & $-0.07,0.10$ & -0.04 & $-0.09,0.01$ & 0.01 & $-0.07,0.09$ \\
\hline Total fat (per unit \% E) & 0.02 & $-0.04,0.08$ & -0.03 & $-0.12,0.07$ & 0.03 & $-0.03,0.09$ & -0.01 & $-0.11,0.09$ \\
\hline SFA (per unit \% E) & -0.05 & $-0.17,0.08$ & -0.18 & $-0.37,0.02$ & -0.02 & $-0.14,0.11$ & -0.12 & $-0.32,0.07$ \\
\hline PUFA (per unit \% E) & 0.01 & $-0.20,0.22$ & -0.03 & $-0.37,0.31$ & -0.003 & $-0.21,0.20$ & -0.02 & $-0.36,0.31$ \\
\hline$P: S$ ratio (per 0.1 unit) & 0.05 & $-0.14,0.24$ & $0 \cdot 21$ & $-0.08,0.51$ & 0.004 & $-0.19,0.20$ & $0 \cdot 14$ & $-0.14,0.43$ \\
\hline
\end{tabular}

$\% E$, energy percentage; $P: S$, PUFA:SFA.

${ }^{*} P<0.05$.

† Estimates adjusted for age, sex, country and BMI.

\section{Discussion}

In the present large multisite cross-sectional study of African origin population samples, we found a strong independent association between increasing protein and total fat intakes and the relative risk of type 2 diabetes across all sites. In both linear and logistic regression models, there was a weaker but significant inverse association between increasing P:S ratio and type 2 diabetes as well as fasting and $2 \mathrm{~h}$ postprandial glucose, for all sites combined. These results emerged despite complex interactions of factors at each site.

As expected, BMI, age and geographic location (compared with Cameroon) were independently associated with increased odds of IFG/IGT and type 2 diabetes. Although BMI was lower in Jamaican men and similar in Jamaican women compared with Cameroon, the levels of $2 \mathrm{~h}$ postprandial glucose and rates of type 2 diabetes were significantly higher, indicating that factors other than adiposity are important in determining glucose handling. Protein and carbohydrate intakes as percentage of total energy were higher in both Jamaica and the UK, which also had higher levels of $2 \mathrm{~h}$ glucose and rates of type 2 diabetes.

While studies in non-diabetic subjects have linked high fat intakes, primarily a high intake of saturated fat (SFA), with an increased risk of $\mathrm{CHD}^{(37-45)}$, the epidemiological evidence linking dietary fat with type 2 diabetes has been inconsistent. Dietary fat is, however, of particular interest because fatty acids influence glucose metabolism by altering cell membrane function, enzyme activity, insulin signalling and gene expression ${ }^{(46)}$. High intakes of SFA may induce insulin resistance and thus worsen glycaemic control $^{(47)}$. Improvements in glycaemia as well as dyslipidaemia have been found with increased consumption of low glycaemic index foods in metabolic studies ${ }^{(48)}$. In populations, generally at higher risk of insulin resistance, such as the US population, it is suggested that diets with increased levels of unsaturated fatty acids, particularly MUFA, have been suggested to have several advantages over high-carbohydrate intakes and may lower the prevalence of diabetes ${ }^{(49)}$. In contrast, a recent European study of carbohydrate intake and the incidence of type 2 diabetes found that a higher carbohydrate intake at the expense of protein and PUFA might be linked to a reduction in diabetes risk ${ }^{(50)}$.

Data from the Iowa Women's Health Study have suggested that the composition of dietary fat intake may play a role in the development of diabetes with an inverse relationship between fat subtype and type 2 diabetes ${ }^{(51)}$. Self-reported dietary intakes generally suggest an inverse association between $n-6$ PUFA intake and diabetes risk $^{(52)}$. Furthermore, as we found here, the European Prospective Investigation of Cancer-Norfolk study reported that a higher energy-adjusted dietary polyunsaturated:saturated fat ratio (P:S ratio) was associated with a reduced risk 
of type 2 diabetes, independent of age, sex, family history of diabetes and other lifestyle factors ${ }^{(9)}$. The present findings are broadly in agreement with this. Taken together, the evidence from published studies suggests that replacing saturated fats and trans-fatty acids with unsaturated (polyunsaturated and/or monounsaturated) fats has beneficial effects on insulin sensitivity and is likely to reduce the risk of type 2 diabetes ${ }^{(46)}$. Among polyunsaturated fats, linoleic acid from the $n-6$ series has been shown to improve insulin sensitivity. This contrasts with data from clinical trials of low-carbohydrate diets that contain a greater proportion of saturated fat, yet resulting in improvement of glucose and lipid control even among persons with type 2 diabetes ${ }^{(53,54)}$

Previously reported results from the present study population have indicated that the habitual diet in rural Cameroon contains more fat and alcohol than the diet in urban Cameroon. Much higher levels of physical activity in the rural subsistence farming community together with lower BMI may explain the lower levels of the cardiovascular risk factors in this area compared to those of the urban dwellers ${ }^{(22)}$. Parallel data show that energy balance rather than diets high in fat per se is a primary cause of excess body fat in Western society ${ }^{(42)}$.

Differences in the association between nutrient intakes and dysglycaemia may be a reflection of the habitual dietary profiles for the different populations investigated here ${ }^{(29)}$. Foods containing carbohydrates, primarily from rice and peas, bread, green bananas and mangoes, were important contributors to reported energy intake in Jamaica. Cassava contributed $44 \%$ of the carbohydrate energy intake in rural Cameroon. The inverse relationship observed between carbohydrate and glucose levels in the Jamaican participants may be a consequence of the proportion of dietary fibre ${ }^{(55)}$, fruits ${ }^{(56)}$, vitamins and antioxidants, a marker of a healthy diet and a negatively correlated glycaemic index ${ }^{(57)}$. In contrast, fats primarily from animal sources, in Jamaica but more so in the UK adults, adversely affect glucose metabolism, and are reported to increase diabetes incidence, independent of obesity and lifestyle factors ${ }^{(51)}$. Nuts (rich in fibre and $\mathrm{Mg}$ ) contributed 5 and $12 \%$ of energy from saturated fat and polyunsaturated fat to the higher reported energy intake in the Cameroonian respondents (who had the lowest rate of dysglycaemia), supporting the results of a meta-analysis of prospective studies which showed that an increased consumption of Mg-rich foods such as whole grains, beans, nuts and green leafy vegetables may reduce the risk of type 2 diabetes ${ }^{(58)}$.

The mechanisms mediating the effect of dietary macronutrients on insulin resistance and glucose handling are complex. It may be the case that protein intake serves as a marker for foods which are diabetogenic. It has been argued that while protein does not contribute to sustained elevations of glucose levels nor slow absorption of carbohydrate, it is just as potent a stimulant of insulin secretion as glucose ${ }^{(59)}$. A higher protein intake may be a marker of a more affluent lifestyle with attendant lower activity levels.

The findings of the present study accord with those of the Nurses' Health Study, which demonstrated an increase in the risk of diabetes with increasing red and other processed meat intake during 14 years of follow-up ${ }^{(60)}$. A similar association was also demonstrated in the Women's Health Study where the significantly increased diabetes risk appeared to be most pronounced for frequent consumption of total processed meat ${ }^{(61)}$. These results remained significant after further adjustment for intakes of dietary fibre, glycaemic load and total fat. More recently, the European Prospective Investigation into Cancer and Nutrition study demonstrated that diabetes risk increased with higher total protein and animal protein intakes at the expense of percentage energy from carbohydrates or fat ${ }^{(62)}$.

\section{Study limitations}

This is a cross-sectional study and, as such, is subject to a number of biases or confounding factors. As with all cross-sectional studies, the temporal ordering among the relationships observed cannot be established. Physical activity, an important determinant of energy expenditure, was not measured in the present study. Another potential limitation of the present study is the lack of adjustment for the effects of lifetime exposure to urban environment and recent migration or current residence, which have been identified as risk factors for obesity and diabetes mellitus ${ }^{(63)}$. Estimates of dietary intakes are obtained from QFFQ that have the potential for measurement error, and respondents are known to under-report dietary intakes (although energy intakes reported here remained high). It is possible that there are other confounders that were not examined in the present study that could also lead to residual confounding.

\section{Conclusions}

Diet energy contributed by specific macronutrients may contribute to IFG/IGT and diabetes prevalence between and within African origin population sites. In multivariate analyses, each unit increase in energy from protein and fats was associated with a increased risk of type 2 diabetes, while an increased P:S ratio was associated with a reduction in risk. These independent effects of dietary factors, within genetically similar groups at different stages of the nutrition transition, are further evidence for modifiable lifestyle effects on impaired glucose handling. The study highlights the potential for specific nutritional components of diabetes prevention programmes in at-risk populations worldwide. 


\section{Acknowledgements}

S. G. A. and N. Y. participated in the study analysis, interpretation of data, drafting of the manuscript and provided statistical expertise. M. K. T.-R., A. H. H. and W. P. S. participated in the interpretation of data and critical revision of the manuscript. J. K. C., R. W., T. F., B. B., J.-C. M., M. D. J., A.T. and S.S. participated in the study concept and design, acquisition of data, interpretation of data and critical revision of the manuscript. All the authors provided input to the manuscript, and none of the authors had any conflict of financial or personal interest. S. G. A. is currently an NIHR Academic Clinical Fellow in Cardiovascular Medicine and was supported as a medical student by a BNF Travel Award and a Denis Burkit Travel Award from the British Nutrition Foundation. The present study was supported by a grant from the European Commission no. TS3*CT92-0142. We would like to thank the Project Staff and all respondents for their participation in the present study.

\section{References}

1. Rich SS, Norris JM \& Rotter JI (2008) Genes associated with risk of type 2 diabetes identified by a candidate-wide association scan: as a trickle becomes a flood. Diabetes $\mathbf{5 7}$, 2915-2917.

2. van Hoek M, Dehghan A, Witteman JC, et al. (2008) Predicting type 2 diabetes based on polymorphisms from genomewide association studies: a population-based study. Diabetes 57, 3122-3128.

3. Lyssenko V, Jonsson A, Almgren P, et al. (2008) Clinical risk factors, DNA variants, and the development of type 2 diabetes. $N$ Engl J Med 359, 2220-2232.

4. Meigs JB, Shrader P, Sullivan LM, et al. (2008) Genotype score in addition to common risk factors for prediction of type 2 diabetes. $N$ Engl J Med 359, 2208-2219.

5. Heald AH, Cade JE, Cruickshank JK, et al. (2003) The influence of dietary intake on the insulin-like growth factor (IGF) system across three ethnic groups: a population-based study. Public Health Nutr 6, 175-180.

6. Sandhu MS, Gibson JM, Heald AH, et al. (2003) Low circulating IGF-II concentrations predict weight gain and obesity in humans. Diabetes 52, 1403-1408.

7. Lastra G, Manrique CM \& Hayden MR (2006) The role of beta-cell dysfunction in the cardiometabolic syndrome. J Cardiometab Syndr 1, 41-46.

8. Boden G \& Laakso M (2004) Lipids and glucose in type 2 diabetes: what is the cause and effect? Diabetes Care $\mathbf{2 7}$, 2253-2259.

9. Harding AH, Day NE, Khaw KT, et al. (2004) Dietary fat and the risk of clinical type 2 diabetes: the European prospective investigation of Cancer-Norfolk study. Am J Epidemiol 159, $73-82$.

10. Mensink RP, Aro A, Den Hond E, et al. (2003) PASSCLAIM Diet-related cardiovascular disease. Eur J Nutr 42, Suppl. 1, I6-I27.

11. Fischbacher CM, Hunt S \& Alexander L (2004) How physically active are South Asians in the United Kingdom?. A literature review. J Public Health (Oxf) 26, 250-258.

12. Mbanya JC, Cruickshank JK, Forrester T, et al. (1999) Standardized comparison of glucose intolerance in west African-origin populations of rural and urban Cameroon,
Jamaica, and Caribbean migrants to Britain. Diabetes Care 22, 434-440.

13. Lee CM, Huxley RR, Lam TH, et al. (2007) Prevalence of diabetes mellitus and population attributable fractions for coronary heart disease and stroke mortality in the WHO South-East Asia and Western Pacific regions. Asia Pac J Clin Nutr 16, 187-192.

14. Magliano D, Söderberg S, Zimmet P, et al. (2010) Mortality, all-cause and CVD, over 15 years in multi-ethnic Mauritius: the impact of diabetes and intermediate forms of glucose tolerance. Diabetes Care 8; (Epublication ahead of print version 8 June 2010).

15. Mayer-Davis EJ, Sparks KC, Hirst K, et al. (2004) Dietary intake in the diabetes prevention program cohort: baseline and 1-year post randomization. Ann Epidemiol 14, 763-772.

16. Knowler WC, Barrett-Connor E, Fowler SE, et al. (2002) Reduction in the incidence of type 2 diabetes with lifestyle intervention or metformin. $N$ Engl J Med 346, 393-403.

17. Pedersen $O$ \& Gaede $P$ (2003) Intensified multifactorial intervention and cardiovascular outcome in type 2 diabetes: the Steno-2 study. Metabolism 52, 19-23.

18. Jackson M, Walker S, Cruickshank JK, et al. (2007) Diet and overweight and obesity in populations of African origin: Cameroon, Jamaica and the UK. Public Health Nutr 10, $122-130$.

19. Mennen LI, Jackson M, Sharma S, et al. (2001) Habitual diet in four populations of African origin: a descriptive paper on nutrient intakes in rural and urban Cameroon, Jamaica and Caribbean migrants in Britain. Public Health Nutr 4 , $765-772$.

20. Sharma S, Cade J, Landman J, et al. (2002) Assessing the diet of the British African-Caribbean population: frequency of consumption of foods and food portion sizes. Int $J$ Food Sci Nutr 53, 439-444.

21. Jackson M, Walker S, Cade J, et al. (2001) Reproducibility and validity of a quantitative food frequency questionnaire among Jamaicans of African origin. Pub Health Nutr 4, 971-980.

22. Mennen LI, Mbanya JC, Cade J, et al. (2000) The habitual diet in rural and urban Cameroon. Eur J Clin Nutr 54, 150-154.

23. Mennen LI, Jackson M, Cade J, et al. (2000) Underreporting of energy intake in four populations of African origin. Int J Obes Relat Metab Disord 24, 882-887.

24. Cooper R, Rotimi C, Ataman S, et al. (1997) The prevalence of hypertension in seven populations of west African origin. Am J Public Health 87, 160-168.

25. Cooper RS, Rotimi CN, Kaufman JS, et al. (1997) Prevalence of NIDDM among populations of the African diaspora. Diabetes Care 20, 343-348.

26. Cruickshank JK, Mbanya JC, Wilks R, et al. (2001) Hypertension in four African-origin populations: current 'Rule of Halves', quality of blood pressure control and attributable risk of cardiovascular disease. J Hypertens 19, 41-46.

27. Cruickshank JK, Mbanya JC, Wilks R, et al. (2001) Sick genes, sick individuals or sick populations with chronic disease? The emergence of diabetes and high blood pressure in African-origin populations. Int J Epidemiol 30, 111-117.

28. Luke A, Durazo-Arvizu R, Rotimi C, et al. (1997) Relation between body mass index and body fat in black population samples from Nigeria, Jamaica, and the United States. $A m \mathrm{~J}$ Epidemiol 145, 620-628.

29. Sharma S, Cade J, Jackson M, et al. (1996) Development of food frequency questionnaires in three population samples of African origin from Cameroon, Jamaica and Caribbean migrants to the UK. Eur J Clin Nutr 50, 479-486.

30. Sharma S, Mbanya JC, Cruickshank K, et al. (2007) Nutritional composition of commonly consumed composite 
dishes from the Central Province of Cameroon. Int J Food Sci Nutr 58, 475-485.

31. Sharma S, Yacavone MM, Cao X, et al. (2009) Nutritional composition of commonly consumed composite dishes for Afro-Caribbeans (mainly Jamaicans) in the United Kingdom. Int J Food Sci Nutr 60, 140-150.

32. Tan S, Wenlock R \& Buss D (1985) Immigrant Foods: Second Supplement to McCance and Widdowson's The composition of foods. London: HMSO.

33. Ngosom J \& Abono A (1989) Les resources alimentaires du Cameroun: Répartition Ecologique, classification et valeur nutritive (The Food Resources of Cameroon: Ecological Distribution, Classification and Nutritional Value). Yaoundé: Institut de Recherche Médicinale et d'études de plantes médicinales.

34. Holland B, Welch A, Unwin I, et al. (1991) McCance and Widdowson's The Composition of Foods. London: The Royal Society of Chemistry.

35. Fletcher L (1994) Microdiet. Salford: University of Salford.

36. Willett WC, Howe GR \& Kushi LH (1997) Adjustment for total energy intake in epidemiologic studies. Am J Clin Nutr $\mathbf{6 5}$, 1220S-1228S; discussion 1229S-1231S.

37. Keys A (1975) Coronary heart disease-the global picture. Atherosclerosis 22, 149-192.

38. Kuulasmaa K, Tunstall-Pedoe H, Dobson A, et al. (2000) Estimation of contribution of changes in classic risk factors to trends in coronary-event rates across the WHO MONICA Project populations. Lancet 355, 675-687.

39. Sanderson P, Gill JM, Packard CJ, et al. (2002) UK Food Standards Agency cis-monounsaturated fatty acid workshop report. Br J Nutr 88, 99-104.

40. Ascherio A (2002) Epidemiologic studies on dietary fats and coronary heart disease. Am J Med 113, Suppl. 9B, 9S-12S.

41. Arnett DK, McGovern PG, Jacobs DR, et al. (2002) Fifteenyear trends in cardiovascular risk factors (1980-1982 through 1995-1997): the Minnesota Heart Survey. Am J Epidemiol 156, 929-935.

42. Willett WC \& Leibel RL (2002) Dietary fat is not a major determinant of body fat. Am J Med 113, Suppl. 9B, 47S-59S.

43. Eberly LE, Stamler J \& Neaton JD (2003) Relation of triglyceride levels, fasting and nonfasting, to fatal and nonfatal coronary heart disease. Arch Intern Med 163, 1077-1083.

44. Warensjo E, Sundstrom J, Vessby B, et al. (2008) Markers of dietary fat quality and fatty acid desaturation as predictors of total and cardiovascular mortality: a population-based prospective study. Am J Clin Nutr 88, 203-209.

45. Anderson SG, Sanders TA \& Cruickshank JK (2009) Plasma fatty acid composition as a predictor of arterial stiffness and mortality. Hypertension 53, 839-845.

46. Risérus U, Willett WC \& Hu FB (2009) Dietary fats and prevention of type 2 diabetes. Prog Lipid Res 48, 44-51.

47. Louheranta AM, Schwab US, Sarkkinen ES, et al. (2000) Insulin sensitivity after a reduced-fat diet and a monoeneenriched diet in subjects with elevated serum cholesterol and triglyceride concentrations. Nutr Metab Cardiovasc Dis 10, $177-187$

48. Liu S \& Willett WC (2002) Dietary glycemic load and atherothrombotic risk. Curr Atheroscler Rep 4, 454-461.

49. Grundy SM, Abate N \& Chandalia M (2002) Diet composition and the metabolic syndrome: what is the optimal fat intake? Am J Med 113, Suppl. 9B, 25S-29S.

50. Schulze MB, Schulz M, Heidemann C, et al. (2008) Carbohydrate intake and incidence of type 2 diabetes in the European Prospective Investigation into Cancer and Nutrition (EPIC)-Potsdam Study. Br J Nutr 99, 1107-1116.

51. Meyer KA, Kushi LH, Jacobs DR, et al. (2001) Dietary fat and incidence of type 2 diabetes in older Iowa women. Diabetes Care 24, 1528-1535.

52. Hu FB, van Dam RM \& Liu S (2001) Diet and risk of Type II diabetes: the role of types of fat and carbohydrate. Diabetologia 44, 805-817.

53. Summers LK, Fielding BA, Bradshaw HA, et al. (2002) Substituting dietary saturated fat with polyunsaturated fat changes abdominal fat distribution and improves insulin sensitivity. Diabetologia 45, 369-377.

54. O' Neill D, Westman EC \& Bernstein RK (2003) The effects of a low-carbohydrate regimen on glycemic control and serum lipids in diabetes mellitus. Metab Syndr Relat Disord 1, 291-298.

55. Liu S, Manson JE, Stampfer MJ, et al. (2000) A prospective study of whole-grain intake and risk of type 2 diabetes mellitus in US women. Am J Public Health 90, 1409-1415.

56. Liu S, Manson JE, Lee IM, et al. (2000) Fruit and vegetable intake and risk of cardiovascular disease: the Women's Health Study. Am J Clin Nutr 72, 922-928.

57. Liu S, Willett WC, Stampfer MJ, et al. (2000) A prospective study of dietary glycemic load, carbohydrate intake, and risk of coronary heart disease in US women. Am J Clin Nutr 71, 1455-1461.

58. Larsson SC \& Wolk A (2007) Magnesium intake and risk of type 2 diabetes: a meta-analysis. J Intern Med 262, 208-214.

59. Franz MJ (2002) Protein and diabetes: much advice, little research. Curr Diab Rep 2, 457-464.

60. Fung TT, Schulze M, Manson JE, et al. (2004) Dietary patterns, meat intake, and the risk of type 2 diabetes in women. Arch Intern Med 164, 2235-2240.

61. Song Y, Manson JE, Buring JE, et al. (2004) A prospective study of red meat consumption and type 2 diabetes in middle-aged and elderly women: the women's health study. Diabetes Care 27, 2108-2115.

62. Sluijs I, Beulens JW, van der AD, et al. (2009) Dietary intake of total, animal, and vegetable protein and risk of type 2 diabetes in the European Prospective Investigation into Cancer and Nutrition (EPIC)-NL study. Diabetes Care 33, 43-48.

63. Sobngwi E, Mbanya JC, Unwin NC, et al. (2004) Exposure over the life course to an urban environment and its relation with obesity, diabetes, and hypertension in rural and urban Cameroon. Int J Epidemiol 33, 769-776. 